\title{
Neuere Entscheidungen zum Stromzahlungsboykott Dokumentation
}

Seit 1977 praktizieren Atomkraftgegener in der Bundesrepublik eine bis dahin unübliche Protestform gegen Atomlagen: den teilweisen Boykott von Stromzahlungen, kurz Strobo genannt. Sie kürzen die Stromrechnungen um einen Betrag von zehn Prozent, den sie auf ein (meist anwaltliches) Treuhandkonto überweisen. D. h., sic verweigern nicht kategorisch die Gegenleistung für den verbrauchten Strom, sondern halten den Teil zurück, der den Atomstromanteil symbolisieren soll. Den schätzungsweise 8000 Teilnehmern dieser Aktion geht es zum einen darum, etwas gegen die Umwelt und Leben bedrohende Nutzung von Atomenergie zu unternehmen; ihr Protest richtet sich dabei unmittelbar gegen die Profiteure der gegenwärtigen Energieversorgung - in aller Regel die Elektrizitätsversorgungsunternehmen (EVU) -. Zum anderen betrachten die Stromzahlungsboykotteure ihren mit der Aktion zum Ausdruck gebrachten Protest als notwendige und legitime Form des *zivilen Ungehorsams «" ${ }^{1}$, um auf Mißstände in der Daseinsvorsorge des Staates hinzuweisen und um eine für unumgänglich und dringend erachtete energiepolitische Wende zu bewirken, die von den Kommunen bzw. vom Geserzgeber bisher noch nicht in Angriff genommen wurde. Deshalb halten sich die Atomstromgegner auch nicht an die üblichen Protestformen und Petitionsmöglichkeiten, die das parlamentarische System bereitstellt, sondern machen die Justiz gewissermaßen zur "Dritten Kammer «, die ihren schutzwürdigen Interessen Geltung verschaffen soll. Ohne auf die mit dieser Strategie - die im übrigen nicht nur auf justizielle Durchsetzbarkeit abzielt ${ }^{2}$ - aufgeworfenen Fragen nach den Möglichkeiten und Grenzen des Schutzes von Minderheitsinteressen gegen parlamentarische Mehrheitsentscheidungen systematisch einzugehen, werden im folgenden neuere Tendenzen der Rechtsprechung zum Stromzahlungsboykott dokumentiert.

Das Urteil des Amtsgerichts Stuttgart vom 20.7.1979 hat als erste positive Entscheidung dem Strobo überraschend großen Auftrieb gegeben. Ungeachtet einer Fülle anderslautiger Urteile, in denen jeder rechtlich relevante Zusammenhang zwischen der Herstellung von Strom in Atomkraftwerken (AKW) und der Rechnungsforderung des EVU abgelehnt wird, *boykottieren ${ }^{\prime}$ ' immer mehr Atomkraftgegner die volle Bezahlung der Stromrechnung. Ähnlich bedeutsam wird ein jüngeres, noch unveröffentlichtes Urteil des AG Gelsenkirchen-Buer sein, nach welchem eine evangelische Kirchengemeinde zur Kürzung der Stromrechnung aus Gewissensgründen berechtigt ist, wenn ihr atomar erzeugter Strom geliefert wird.

1 Zur Geschichte und zum Begriff des Zivilen Ungehorsans vgl. Wulfgang Zucht, Der Zivile Ungehorsam, in: Kein Atomkraftwerk mit unserem Geld (hrsg. v. T. Hengesbach/M. Schweitzer, 2. Aufl. Dortmund $197^{8}$ ), S. 17 ff.

2 W. Rülle und T. Hengesbach schreiben dazu in ihrer Broschüre Stromgeldverweigerung - Perspektiven und künfeige Probleme (Dortmund r 979), S. 6: . Unter Zivilem Ungehorsam verstehen wir die bewußte, gewaltlose und öffentliche Verletzung von Gesetzen und geserzesähnlichen Bestimmungen zum Zwecke des Protestes, wobei man sich nicht den dafür vorgesehenen Strafen oder Folgen zu entzichen sucht.* 3 Abgedruckt findet sich die Entscheidung in NIW 1979, S. 2047 f.

4 Die Zahl der Urteile kann auf 300 bis 400 geschätzt werden; für die Beschaffung der nachstehend zitierten, sämtlich unveröffentlichten Entscheidungen schuldet der Verfasser dem Strobo-Archiv des Umweltwissenschaftlichen Instituts e.V. in Stuttgart Dank.

5 J. Gerlach, -Stromzahlungsbuykort* - ein sinnvolles Mittel im Kampf für Umweltschutz?, Demokratie und Recht 1980, S. 363, 365 weist darauf hin, daß es sich nicht um einen Boykott im üblichen Sinne handelt. 
Tatbestand: Die beklagte Kirchengemeinde erklärte gegenüber dem klägerischen EVU die Teilnahme am Stromzahlungsboykott und kürzte Stromrechnungen um insgesamt $68_{4}, 6 \mathrm{r}$ DM. Die Klägerin beantragt,

1) Zahlung von $68_{4}, 6 \mathrm{I} \mathrm{DM}$

2) Feststellung, daß die Beklagte aus Stromliefervertrag auch künftig verpflichtet ist, die in Rechnung gestellten Strompreise ungekürzt zu zahlen.

Aus den Entscheidungsgründen:

Der Zahlungsantrag ist begründet; der Feststellungsantrag ist zulässig, jedoch nicht vollständig begründet.

I. Zum Feststellungsantrag

Der Antrag der Klägerin ist gemäß $\$ 256 \mathrm{ZPO}$ zulässig ...

Der Feststellungsantrag ist jedoch nicht vollständig begründet. Grundsätzlich hat die Beklagte, was zwischen den Partcien auch unstreitig ist, die Stromrechnungen der Klägerin analog $\ 433$ Abs. 2 BGB in Verbindung mit der Verordnung über die allgemeinen Bedingungen für die Elektrizitätsversorgung von Tarifkunden vom 21.6.79 (AVBEltV) zu zahlen.

Dieser Verpflichtung der Beklagten steht jedoch, soweit die Klägerin die Beklagte in Zukunft mit Strom versorgen sollte, welcher aus der Produktion von Atomkraftwerken stammt, ein Leistungsverweigerungsrecht in Höhe des Anteils des gelieferten *Atomstroms« zu. Dieses Gegenrecht beruht auf $\$ 242$ BGB in Verbindung mit Art. ${ }_{4}$ Abs. I GG.

Die Beklagte ist auch berechtigt, enrweder direkt oder über $\$ 242$ BGB ihre religiös begründete Gewissensentscheidung unter Berufung auf Art. ${ }_{4} \mathrm{GG}$ in das zwischen der Klägerin und der Beklagten bestehende privatrechtliche Dauerschuidverhältnis einzubringen. Zum einen ist weitgehend anerkannt und wird vom Gericht ebenfalls so gesehen, daß Gewissensentscheidungen, und zwar grundsätzlich ohne Rücksicht auf ihren Inhalt, über $\$ 242$ BGB Auswirkungen auf das Privatrecht und bestehende Vertragsverpflichtungen haben können (siehe z. B. Larenz, Schuldrecht Bd. I, 12. Aufl. 79, \$10 IIc); zum anderen nimmt die Klägerin als privatrechtlich organisiertes Unternehmen Aufgaben der Energieversorgung wahr, die gemäß $\{2$ Abs. 2 Energiewirtschaftsgesetz (EnWG) ohne Rücksicht auf die Rechtsform der Klägerin als öffentlich anzusehen sind mit der Folge, daß auch eine direkte Anwendung des Art. 4 Abs. 1 GG möglich ist (siehe dazu Hermann in BB $79 / 602$ mit weiteren Nachweisen).

Das Gericht geht auch davon aus, daß sich die Beklagte aufgrund ihres religiös begründeten Gewissens nicht in der Lage sieht, den anfallenden Strompreis vollständig zu zahlen. Dies ergibt sich sowohl aus den überzeugenden Ausführungen der Beklagten zur Frage ihrer Gewissenlage im Klageerwiderungsschriftsatz vom I6. 2. 8 I als auch den mündlichen Ausführungen, die die Mitglieder des Presbyteriums der Beklagten im Termin vom 30. 3. 81 gemacht haben. Danach besteht zwischen den Gefahren und Risiken der Nutzung von Atomenergie und den Bekenntnisgrundsätzen der Mitglieder der Beklagten als Evangelische Christen ein so starker Widerspruch, daß sich die Beklagte nicht mehr in der Lage sieht, den weiteren Ausbau und die weitere Nutzung von Atomenergie in irgendeiner Form zu unterstützen.

Die Gewissensnotlage berechtigt die Beklagte, im Rahmen des zwischen ihr und der Klägerin bestehenden Dauerschuldverhältnisses von der Klägerin zu veriangen, keinen atomar erzeugten Strom an die Beklagte zu liefern und gegebenenfalls in Höhe des gelieferten Atomstromanteils den Kaufpreis einzubehaiten.

Zwar ist in Rechtsprechung und Literatur in erster Linie nur anerkannt, daß bei Dauerschuldverhältnissen die Gewissensnot des einen Vertragspartners möglicherweise zum außerordentlichen Kündigungsrecht aus wichtigem Grund berechtigt; dies ist jedoch vorliegend kein ausreichendes und geeignetes Mittel, der Gewissensnot der Beklagten abzuhelfen; denn die Beklagte ist wie jeder andere Bürger auf die Versorgung mit elektrischer Energie angewiesen und ihr steht keine Möglichkeit zu, auf andere Weise als durch Lieferung seitens der Klägerin dieses berechtigte Bedürfnis zu erfüllen. Die Klägerin nimme für den Bereich der Beklagten hinsichtlich der Versorgung mit elektrischer Energie eine Monopolstellung ein. Dies hat zur Folge, daß die Beklagte ihren Bedarf an elektrischer Energie lediglich durch Lieferungsvertrag mit der Klägerin erfüllen kann. Im Gegensatz zu vielen Gerichten, die in ähnlichen Fällen dem Schuldner auferlegt haben, infolge der Gewissensentscheidung auf jegliche Versorgung mit Stromenergie zu verzichten, ist das Gericht der Auffassung, daß dieser Weg der Beklagten nicht zumutbar ist. Denn dic Beklagte hat einen Anspruch auf Versorgung mit elektrischer Energie im Rahmen der bestehenden Versorgungskapazitäten lediglich aufgrund der im "Dritten Reich begründeten monopolarigen Struktur der Energieversorgung in der Bundesrepublik Deutschland hat die Beklagte keine Möglichkeit, durch Vertragsschluß mit anderen Energieversorgern entsprechend ihrer Gewissenentscheidung zu handeln. 
Durch dieses Gegenrecht wird die Klägerin auch nicht unzumutbar beeinträchtigt. Im Rahmen der vorzunehmenden Güterabwägung zwischen dem Gewissen der Beklagten und den Interessen der Klägerin ist zu bedenken, daß nach der inzwischen unstreitigen Darlegung der KJägerin die Beklagte auch in der Vergangenheit lediglich mit Strom versorgt worden ist, der herkömmlich hergestellt ist, also nicht aus der Produktion von Atomkraftwerken stammt, da das Gebiet Gelsenkirchen-Buer-Hassel ausschließlich mit Strom aus den umliegenden Steinkohle-, Oel- bzw. Braunkohlekraftwerken versorgt wird.

Neben der Monopolstellung der Klägerin und der Tatsache, daß die monopolartige Versorgungsstruktur im Bereich der elektrischen Energieversorgung dor Beklagten nicht anzulasten ist, was nach Auffassung des Gerichts der entscheidende Grund dafür ist, daß die Beklagte entsprechend ihrer Gewissensentscheidung berechtigt ist, die Lieferung von atomfreien Strom zu verlangen bzw. gegebenenfalls einen Einbehalt vorzunehmen, ist bei der Rechtsgüterabwägung $z$ wischen den Interessen der Klägerin und dem Gewissensrecht der Beklagten dem letzteren auch deshalb der Vorrang zu geben, weil dem weiteren Betrieb von Kernkraftwerken nach Auffassung des Gerichts erhebliche verfassungsrechtliche Bedenken entgegenstehen. Diese Bedenken beruhen unter anderem darauf, daß trotz der immer noch ungelösten Frage der Entsorgung von AKW die bisher installierten und genehmigten AKW weiter produzieren.

Die Frage der Entsorgung ist im Atomgesetz (AtomG) ausdrücklich erst mit Einführung des $\int 9$ a AtomG am 30. 8. 76 geregelt worden. Eine Rückwirkung dieser Vorschrift auf die bereits vorher genehmigten Kernkraftwerke ist im Gesetz nicht angeordnet und vom Gesetzgeber auch nicht gewollt. Vor Inkrafttreten des $\$ 9$ atomG war die Entsorgungsfrage nach einhelliger Meinung von der Genehmigungsbehörde im Rahmen des $\>$ Abs. 2 Nr. 3 AtomG als Teil ihrer Ermessensübung zu berücksichtigen. Es herrscht zwar für die Zeit nach Inkraftereten des $\$$ ga AtomG in Rechtsprechung und Literatur Streit, ob der Nachweis einer gesicherten Entsorgung nunmehr Genehmigungsvoraussetzung ist oder nach wie vor nur im Rahmen des der Behörde eingeräumten Ermessens, die Genehmigung trotz Erfüllung der Voraussetzungen des $\$ 7$ Abs. 2 Nr. 3 AtomG zu versagen, beachtet werden muß; dies ist im Ergebnis für die vorliegende Beurteilung jedoch nicht von Bedeutung, da nach Auffassung des Gerichts die Genehmigung eines AKW trotz fehlender Entsorgung einen Ermessenfehlgebrauch darstellen würde. Die Frage der Entsorgung ist jedoch zur Zeit als nicht gelöst anzusehen. Nach wie vor ist nicht endgültig klar, ob Salzstöcke für eine Lagerung von atomar verseuchten Abfällen geeignet sind. Hinzu kommt die Notwendigkeit einer ständigen Uberwachung sowohl der stillgelegten Kraftwerke als auch der Endlagerstätten, die kein Staat auch nur für die nächsten 100 Jahre garantieren kann, obwohl unstreitig die Notwendigkeit einer gewissen Kontrolle der Endlagerstätten mindestens für die nächsten 1000 Jahre gegeben ist. (Siehe Hofmann *Langzeitrisiko und Verfassung* in Scheidewege I980 Seite 450, 475). Durch die weitere Produktion an hochradioaktivem Abfall wird angesichts der ungelösten Entsorgungsfrage gegen die Grundrechte aus Art. 2 A bs. 2 GG auf das Leben und körperliche Unversehrtheit der zur Zeit lebenden Bürger und der späteren Generationen, zu deren Schutz der Staat, wie sich aus Art. I Abs. I GG und der Präambel des Grundgesetzes ergibt (siehe im einzelnen Hofmann a. a. O. 45 I ff.), ebenfalls verpflichtet ist, verstoßen (siehe Hofmann a. a. O., 477; Gleim, Winter in NJW 80/1089).

Dieser Gefährdungseingriff in die Rechte der lebenden Grundrechtsträger wäre allenfalls zulässig, wenn er zum Schutz überragender Gemeinwohlgüter zwingend erforderlich wäre, wovon nicht auszugehen ist.

Soweit die Grundrechte zukünftiger Generationen herangezogen werden, wobei fraglich ist, ob überhaupt eine Güterabwägung in üblicherweise wegen der Nichtidentität von NutznieBern und Gefährdeten zulässig ist, gilt das gleiche.

Denn in Rahmen der vorzunehmenden Verhältnismäßigkeitsprüfung ist zu berücksichtigen, daß eine Notlage mit dem daraus folgenden $Z$ wang zur weiteren Produktion von Kernkraftstrom und zum weiteren Ausbau dieses Produktionszweiges bei ungelöster Frage der Entsorgung solange nicht besteht, wie neue Kohlekraftwerke mit Fernwärmenutzung nicht gebaut werden, die dezentralisierte Kraft-Wärmekopplung vernachlässigt wird, die industrielle Eigenerzeugung von Abwärmestrom durch die regionalen Großunternehmen der Stromerzeugungswirtschaft behindert und zurückgeschraubt wird (siehe Mestmärker, Recht und ökonomisches Geserz, Baden-Baden 1978, Seite 721), staatliche Normen zur Wärmedämmung bei Gebäuden und Isolierung elektrischer Großgeräte nicht verschärft werden und das Tarifsystem der Stromerzeuger zu höherem Stromverbrauch animiert (siehe Hofmann a. a. O., Seite 477 , $478)$.

Diesen oben geschilderten Gegenrechten der Beklagten kann auch nicht entgegengehalten werden, $d a ß$ die Beklagte entsprechend $\$ 6$ Abs. 3 EnWG selbst eine Energieanlage zur 
Erzeugung von Elektrizität betreiben könne. Zum einen ist dem Gericht nicht ersichtlich, wie die Beklagte technisch und wirtschaftlich ein solches Vorhaben überhaupt bewäligen könnte; zum anderen wäre auf jeden Fall eine Zusatzversorgung durch die Klägerin notwendig, die die Beklagte jedoch gemäß $\$ \$ 4,6$ Zif. 6 der s. Durchführungsverordnung zum EnWG nur dann in Anspruch nehmen könnte, wenn die Eigenanlage ausschließlich mit Betriebsabfällen oder mit Wasserkraft betrieben würde, wozu die Beklagte jedoch nicht in der Lage sein dürfte, da sie kein industrielles Unternehmen ist.

Die weitergehenden von der Beklagten infolge ihrer Gewissenentscheidung in Anspruch genommenen Gegenrechte stehen ihr nicht zu. Denn die von der Beklagten in Anspruch genommene Gewissensnotlage berechtigt sie nicht, über den ausgesprochenen Umfang hinaus die Klägerin zu zwingen, auf jegliche Verwendung, Verteilung und Einkauf von atomar erzeugtem Strom zu verzichten oder gar ihre Verragsbeziehungen zum RWE zu beenden. Denn dies hieße, daß die Beklagte mit Hilfe ihrer Gewissensentscheidung das gesamte wirtschaftliche Verhaiten der Klägerin dominieren würde, wozu die Beklagte nicht befugt ist. Selbst wenn ihre Auffassung von der Gefährlichkeit der Nutzung der Atomenergie richtig sein mag, hat sie dennoch nicht das Recht, im Rahmen des bestehenden Vertragsverhältnisses diese Auffassung zugunsten aller Bürger und Stromabnehmer einseitig durchzusetzen.

Auch das von der Beklagten zuletzt in Anspruch genommene Zurückbehaltungsrecht aus $\$ 273$ BGB steht ihr hinsichtlich der oben erwähnten weitergehenden Gegenrechte nicht zu. Einen vertraglichen Anspruch auf Unterlassung der Produktion von Kernkraftstrom könnte die Beklagte allenfalls dann geltend machen, wenn die Klägerin selbst Strom produzieren würde (so für den Fall der Identität zwischen Stromerzeuger und Lieferant AG Stuttgart in NJW 79/2047; anderer Ansicht: Lüke in NJW 79/2049 mit im Stil herablassenden Argumenten und abenteuerlichen Uberlegungen zur Frage der Beseitigung des Urteils; AG Stuttgart in NJW 80/ro08). Lediglich die Lieferung von atomar erzeugtem Strom erhöht jedoch ein schon entstandenes Gefährdungspotential nicht, so daß daraus ein vertragliches Gegenrecht nicht herleitbar ist.

Auch ein deliktischer oder quasinegarorischer Unterlassungsanspruch der Beklagten wegen einer möglichen Verletzung der der Klägerin jedem Mitglied der Allgemeinheit gegenüber obliegenden Pflicht, nicht in Leben und Gesundheit rechtswidrig einzugreifen, kann als Gegenanspruch nicht herangezogen werden im Rahmen des $\$ 273 \mathrm{BGB}$, da es an der erforderlichen $\star$ Konnexität z.wischen dem möglicherweise gegebenen Unterlassungsanspruch und dem Anspruch auf Strompreiszahlung fehlt. Denn die Stromerzeugung wird nicht von der Klägerin, sondern vom RWE vorgenommen. Darüber hinaus bestünden auch Bedenken hinsichtlich der Annahme einer Rechtsverletzung der Beklagten, da diese als juristische Person sich allenfalls auf eine Verletzung ihres Eigentums berufen könnte...

II. Zum Zahlungsantrag

Der Zahlungsantrag der Klägerin ... ist in voller Höhe begründet.

Der Anspruch steht der Klägerin in analoger Anwendung des $\$ 433$ Abs. 2 BGB zu.

Das der Beklagten infolge ihrer Gewissensnot zustehende Gegenrecht, bei Lieferung von atomar erzeugtem Strom Einbehaltung vornehmen zu dürfen, greift vorliegend nicht durch. Denn in dem genannten Zeitraum ist der Beklagten lediglich Strom geliefert worden, der aus der Produktion von herkömmlichen Kohle-, Oel- oder Braunkohlekraftwerken stammt, was unstreitig ist...

Diese Entscheidung ist in mehrfacher Hinsicht bemerkenswert. Dem Gericht gelingt es, ungeachtet eines starken öffentlichen Meinungsdruckes und gegen die herrschende Lehre und Rechtsprechung, eine eigenständige rechtliche Argumentation zu entwickeln. Aus den monopolbedingten Besonderheiten des Stromlieferungsvertrages ${ }^{6}$ wird die Durchschlagkraft des Leistungsverweigerungsrechtes aus Gewissengründen deduziert. Die bei der Bewertung der Frage ebenfalls mitentscheidenden energiepolitischen Vorstellungen des Gerichts werden nicht verschwiegen, sondern offengelegt. Mit diesen auf realer Grundlage fußenden Vorstellungen begeht das Gericht jedoch keine Kompetenzanmaßung hinsichtlich der Entscheidung öffentlich-rechtlicher Fragen; vielmehr wird die diesbezügliche Auffassung des Gerichts, die sich im übrigen auf Beweismaterial der Beklagten stützen kann,

6 R. Knieper, Warum die Atomenergie das Zivilrecht nicht kalt läßt, Kritische Justiz 1980, S. 77, 81 unterstreicht weitere Besonderheiten des Stromversorgungsvertrages und hält das klassische Vertragsmodell nicht für anwendbar; dagegen Gerlach, aaO, S. 380. 
nur zur Entscheidung der Frage herangezogen, ob es der Klägerin zumutbar ist, die Ausübung eines vorhandenen Leistungsverweigerungsrechtes der Beklagten hinzunehmen. Bei der Beantwortung dieser Frage ist aber in der Tat das Vorhandensein wirtschaftlicher Alternativen zur Atomenergie maßgebend. Das Gericht nimmt sich weder das Recht, noch gibt es der Beklagten dieses Recht, über die Wirtschafts- und Energiepolitik des klägerischen EVU zu bestimmen.

Problematisch am Urteil des AG Gelsenkirchen-Buer erscheint indes die Eingrenzung des Leistungsverweigerungsrechtes dahingehend, daß tatsächlich in AKW hergestellter Strom geliefert wird. Die vom Gewissen verbotene Handlung, die Unterstützung der Atomkraft in jeglicher Weise, wird unabhängig davon durch die volle Bezahlung der Stromrechnung bewirkt. Der genehmigungspflichtige Strompreistarif berücksichtigt nämlich Investitionskosten für Kraftwerke; diese Investitionen werden zur Zeit fast ausschließlich im Bereich der Atomenergie getätigt. Auch konstruktive Schwierigkeiten des Gerichts, einen im Rahmen des $\ 273$ BGB relevanten Unterlassungsanspruch zu begründen, hätten vermieden werden können. Wegen der öffentlich-rechtlichen Monopolstruktur der EVU kann jedenfalls eine Identität zwischen Stromerzeuger und Lieferant kaum gefordert werden. Es erscheint zufällig, von welchem EVU - es handelt sich in der Regel um privatrechtlich organisierte Eigenbetriebe der Gebietskörperschaften - der Atomstrom erzeugt wird, den ein anderes EVU liefert. $\$ 278 \mathrm{BGB}$ ist zwischen diesen Unternehmen anwendbar. Im übrigen kommt unter Rückgriff auf $\$ 24_{2}$ BGB die Durchgriffshaftung in Betracht.

Letztlich ist allerdings zu befürchten, daß das vorliegende Urteil mit der vom kJägerischen EVU eingelegten Berufung aufgehoben wird. In der Rechtsprechung herrscht eine Tendenz vor, die Bindungswirkung eines Vertrages in den Vordergrund zu rücken. Daher ist es wenig wahrscheinlich, daß das Berufungsgericht die vom AG Gelsenkirchen-Buer gezogenen Konsequenzen nachvollziehen wird. Diese Tendenz mögen die Auszüge aus zwei anderen Urteilen verdeutlichen:

\section{b. Urteil des LG Stuttgart v. I8. I2. I980, Az.: $100164 / 80$ :}

Diese mittelbare Drittwirkung des Grundrechts führt aber nicht dazu, daß der Beklagte jeweils $10 \%$ der Rechnungsbeträge einbehalten darf. Der Gewissensentscheidung des Beklagten steht eine nicht nur rechtliche, sondern auch sittliche Pflicht gegenüber, Vertragstreue zu üben (Hervorhebung d. V.), d. h., einen geschlossenen Vertrag zu halten. Die Gegenüberstellung dieser sittlichen und auch rechtlichen Normen führt nicht ohne weiteres dazu, daß die Gewissensfreiheit den unbedingten Vorrang vor den vertraglichen Bedingungen hat. Für die Frage, wie sich das vom Schuldner in Anspruch genommene Grundrechr der Gewissenfreiheit zuf seine vertragliche Verpflichtung auswirkt, ist bedeutsam, welchen Inhalt seine Verpflichrung hatte. Die Gewissensfreiheit erhält umsomehr Gewicht, je persönlichkeitsbezogener seine vertragliche Erfüllungsverpflichtung ist. Bei einer Geldschuld kommt bei der Abwägung zwischen 'Gewissensfreiheit des Einzelnen und der Ordnungsfunktion des allgemeinen Rechts der letzteren aus den überwiegenden Gründen des Allgemeinwohls der Vorrang zu. Folgte man der Auffassung des Beklagren, so würde dies zu einer nicht vertretbaren Beschränkung der Freiheitssphäre des Gläubigers dadurch führen, daß man es dem Schuldner überließe, seine Geldleistung von einem von ihm zu bestimmenden Verwendungszweck abhängig zu machen. $\mathrm{Zu}$ beschränken ist dieses Problem nicht nur auf den vorliegenden Sachverhalt. Denkbar ist ohne weiteres, daß z. B. Autofahrer, Passagiere eines Flugzeugs, Käufer von Plastikgegenständen und andere zwar überwiegend freiwillig eine Leistung der Gegenseire in Empfang nehmen, ihrer eigenen Leistungspflicht aber mit dem Hinweis auf Gefahren für die Umwelt und ähnliches nur teilweise oder überhaupt nicht nachkommen.

Die Beweggründe für ein solches Vorgehen mögen noch nachvollziehbar und teilweise auch verständlich sein. Unstreitig kann man wohl ferner auch davon ausgehen, daß die Aktion, wie die des Beklagten, mit dazu geführt hat, die breire Offentlichkeit auf ein gewichtiges Problem aufmerksam zu machen, wobei nicht auszuschließen ist, daß Fragen der Sicherheit bei der 
Errichtung von Kernkraftwerken eine noch größere Bedeutung erlangen. Dies alles kann jedoch nicht dazu führen, den glatten Vertragsbruch (Hervorhebung d. V.) des Beklagten als rechtmäßig zu bezeichnen. Aus den oben genannten Gründen ist dem Grundsatz, Vertragstreue zu üben, Vorrang zu geben vor dem Grundrecht des Artikels 4 Abs. I - vgl. hierzu Urteil des Amtsgerichts Freiburg $2 \mathrm{C} 76 / 80-$.

Das LG Stuttgart zieht offenbar nicht in Betracht, daß der Stromkunde, der mit einem Monopolisten kontrahiert, keineswegs mit dem ^Käufer von Plastikgegenständen* zu vergleichen ist, die Gewissensnot des einen also zu anderen Konsequenzen führen kann und muß, als diejenige des anderen. Deuticher noch zeigt sich ein so kurzschlüssiger Vergleich beim nachfolgenden Urteil:

\section{c. Urteil des AG Hamburg v. I4. II. 1979, Az.: 9 C 774/79:}

In seinen Gewissensentscheidungen ist der Schuldner aber völlig frei, und darum muß er auch das hieraus erwachsene Risiko der Leistungshinderung tragen. Für das, was Saulus versprach, hat Paulus aufzukommen. (Hervorhebung d. V.). Eben weil der Schuldner seine religiöse und weltanschauliche Einstellung jederzeit nach eigener unbeeinflußter Entscheidung wechseln darf, braucht der Gläubiger das hieraus entstehende Gewissensrisiko nicht auf sich zu nehmen. Die Berufung auf das inzwischen geschärfte Gewissen würde übrigens das frühere Versprechen desavoieren, das den anderen zum Vertrag verleitete, ein typischer Fall des venire contra factum proprium. Damit steht das Ergebnis für Vertragspflichten allgemein fest: Der Gewissenskonflikt des Vertragsschuldners hindert eine Leistungserzwingung gegen das Gewissen schlechthin mit der Folge, daß höchstpersönliche Leistungen vom Schuldner nicht zu erbringen sind, der Gewissenskonflikt befreit ihn des Vertrages. Dieses Ergebnis entspricht auch durchaus der Billigkeit: Das Geschäftsleben braucht eine halbwegs klare Abgrenzung des sittlichen Standarts, dem Vertragsschluß und Vertragserfüllung entsprechen müssen, eine Durchschnittsmoral, bei deren Einhaltung Vertragsansprüche gesichert sind. Dann kann keine Partei erwarten, der andere Teil werde zu ihren Gunsten irgendwelchen strengeren ethischen Regein folgen, dann braucht aber auch keine Partei damit zu rechnen, daß ihr Interesse am Vertrag durch eine solche Befolgung beeinträchtigt wird. Uber Vertragspflichten entscheidet das Gewissen des Schuidners zum Schaden des Gläubigers eben nur, wenn es - wenigstens als Bestandteil der Geschäftsgrundlage - zum Schiedsrichter über den Vertrag erhoben wurde. Das ist hier indes nicht der Fall.

Selbst wenn der Kläger (= Stromkunde, d. V.) auf die Monopolstellung der Beklagten hinweist, so berechtigt es ihn nach den dargelegten Gesichtspunkten nicht zu einer Kürzung der Strompauschale. Ein derartiges Verhalten stellt keine Lösung eines Gewissenskonfliktes dar, der Kläger hat diese Lösung vielmehr auf dem politischen Weg oder aber in den dafür vorgesehenen Verfahrenswegen vor den Gerichten zu suchen. Das Festhaiten des Klägers am Strom-Lieferungsvertrag mit der Beklagten macht ihn zugleich zahlungspflichtig, da es - wie Blomeyer, JZ 1954, 309 treffend gesagt hat - nicht der Sinn des Arrikels 4 Abs. I GG ist, sich aus fremder Tasche ein gutes Gewissen zu machen. Wenn die Gewissensnot des Klägers so groß ist, wie er vorgibt, möge er die vertraglichen Beziehungen zur Beklagten gänzlich lösen, denn Gewissensnot ermächtigt zum sofortigen Rücktritt von einem privatrechtlichen Vertrage (vgl. Maunz-Düring, Artikel 4 Randnote 142 mit weiteren Nachweisen).

Bedenklich an diesem Urteil des AG Hamburg erscheint schon das Abstellen auf eine "Durchschnittsmoral «, deren Gewissensentscheidungen allein Einfluß auf den Vertragsinhalt haben soll. Das wird der Bedeutung des Art. 4 Abs. I GG, der jede Gewissensentscheidung schützt, nicht gerecht. Problematischer sind allerdings die Ausführungen zum venire contra factum proprium. Das Amtsgericht geht davon aus, das das EVU auch oder erst dadurch zum Vertragssch]uß *verleitet « worden ist, $\mathrm{da} ß$ der Stromkunde sich kein Gewissen daraus macht, auf welche Art der Strom erzeugt wird. Nach $\$ 6$ EnWG ist das EVU indes zum Vertragsschluß mit jedem Kunden im Versorgungsgebiet verpflichter, unabhängig davon, welche Einstellung der Kunde zur Atomenergie hat. Der Vertragsschluß ist somit Folge eines beiderseitigen Zwanges: weder der Abnehmer kann sich das EVU aussuchen, noch dieses den Abnehmer. Verhält sich der Stromkunde bei „Vertragsschluß gegenüber der Atomenergie gleichgültig, weil er sich mit den damit zusammenhängenden 
Gefahren nicht beschäftigt hat, oder weiß er noch nicht, daß im vorliegenden Fall das hamburgische EVU, die HEW, in großem Ausmaß in der Atomindustrie engagiert ist (und für dieses Engagement von ihren Abnehmern die höchsten Strompreise innerhalb Deutschlands verlangt), so kann dies nicht dem Kunden als venire contra factum proprium angelastet werden. Neben der Sache liegt auch die Uberlegung, der eine Gewissensentscheidung geltendmachende Schuldner müsse jedenfalls Schadensersatz in Geld leisten. Zum einen sind die Schadensersatzregelungen nicht auf eine Geldschuld anwendbar, und zum anderen würde eine solche Verpflichtung in der vorliegenden Konstellation dazu führen, daß der Schuldner gerade das bewirken muß, was ihm sein Gewissen verbietet. Nimmt man daher die grundrechtlich geschützte Gewissensentscheidung ernst, ist der Vertrag entsprechend $\ 242$ BGB bzw. der - anwendbaren - Lehre vom Wegfall der Geschäftsgrundlage der Gewissensentscheidung anzupassen. Ein Verweis auf die Möglichkeit, den Vertrag zu lösen, verbietet sich schon wegen des Fehlens einer anderen Möglichkeit, Strom zu beziehen.

Erwägenswert ist in diesem Zusammenhang noch folgender Aspekt: Die Lösung des AG Hamburg mutet dem eine Gewissensentscheidung geltend machenden Stromzahlungsboykotteur über die Verpflichtung zur Handlung entgegen der Gewissensentscheidung hinaus noch weitere Grundrechtsbeeinträchtigungen zu. Nach den Gegebenheiten des heutigen Lebens erscheint der Entzug der Nutzungsmöglichkeit elektrischer Energie innerhalb der häuslichen Wohnung - konkretisiert dadurch, daß der Gebrauch von Licht, Kühlschrank, Staubsauger, Radio, Fernsehen u. ä. erschwert oder gar unmöglich ist - als Verletzung der Art. 2 Abs. I, 13 Abs. I GG. Eine Güterabwägung, die auch in diesen Fällen, in denen lediglich eine geringfügige, teilweise Vertragsverletzung in Rede steht, dem Vertrag noch Vorrang einräumt, erklärt letztlich die von der Verfassung geschützte Gewissensentscheidung für irrelevant und sanktioniert dies sogar. Interessengerecht erscheint der Konflikt daher nur in der Weise lösbar, daß der Vertrag der Gewissensentscheidung angepaßt werden kann.

Dieser aus der Perspektive der Gewissensfreiheit naheliegenden und im Ansatz vom Amtsgericht Gelsenkirchen-Buer vertretenen Lösungsmöglichkeit stehen natürlich Bedenken entgegen. Im vorstehend zitierten Urteil des Amtsgerichts Hamburg heißt es dazu:

Eine andere Interpretation des Artikels 4 Abs. I GG würde in ihrer Konsequenz dazu führen, daß jeder berechtigt wäre das zu tun, was er für richtig hält, ein durch überindividuelle Normen geordnetes Zusammenleben unmöglich machen und auf $A$ narchie (Hervorhebung d. V.) hinauslaufen.

Es wird befürchtet, daß die Rechtsordnung außerkraft gesetzt wird, wenn jedermann seinen Uberzeugungen gemäß lebte, diese Uberzeugungen also auch rechtlich zu respektierende Wirkungen nach außen zeitigten. Allein, diese Befürchtung ist nicht begründet: die hier vorgeschlagene Konsequenz aus der Gewissensentscheidung gegen Atomkraft ist an enge Voraussetzungen geknüpft und dürfte kaum auf übliche Vertragsverhältnisse übertragbar sein.

Der weitere Einwand, der Stronzahlungsboykotteur mache sich waus fremder Tasche ein gutes Gewissen«, ist in doppelter Hinsicht nicht stichhaltig: Erstens unterstellt er zu Unrecht, daß dieser sich Zahlungen erspare, was gerade nicht zutrifft. Denn Stromzahlungsboykott bedeutet nur, daß die Zweckbestimmung der Gegenleistung hinsichtlich des zurückgehaltenen Teiles verändert werden soll. Es wird allerdings zweitens nicht "einfach “ erfüllt, sondern so erfüllt, daß der Kontrahent veranlaßt werden soll, bei seiner Leistung dem Vertragspartner Gewissensnöte zu ersparen. Da das Eingehen einer vertraglichen Verpflichtung nicht 
automatisch dazu führen kann, nicht vorhergesehene und nicht vorhersehbare und außerdem vermeidbare Gewissensnöte in Kauf nehmen zu müssen - gleichsam als Teil des Kontrakts -, ist vom Vertragspartner zu fordern, daß dieser einem im Rahmen seiner Möglichkeiten solche Gewissensnöte erspart. Dies folgt - wenn nicht schon aufgrund des Monopols des EVU - aus dem öffentlich-rechtlichen Charakter der Elektrizitätsversorgung. Solange ein EVU diese Obliegenheit nicht erfüllt, hat es die dadurch entstehenden Einbußen hinzunehmen. Mit der Annahme einer solchen Obliegenheit wird jedenfalls nicht auf die Unternehmensfreiheit und Energiepolitik des EVU Einfluß genommen: die Obliegenheit läßt sich auch in der Weise erfüllen, daß das Unternehmen für diejenigen Kunden, denen es das Gewissen verbietet, mit der vollen Bezahlung der Stromrechnung die Forcierung der Atomtechnologie zu finanzieren, ein Sonderkonto einrichtet. Den für Investitionen bestimmten Anteil der Stromgelder auf diesem Sonderkonto könnte dann das EVU ausschließlich für den unzweifelhaft volkswirtschaftlich und energiepolitischen Ausbau von sog. alternativen Energiequellen verwenden. Der Gewissensnot des Stromkunden wäre abgeholfen. Gleichzeitig ist die Belastung des EVU denkbar gering, weil Investitionen für »sanfte Energie* schon jetzt, allerdings in bescheidenem Ausmaße, vorgenommen werden. ${ }^{7}$

Nach der Erörterung des Leistungsverweigerungsrechtes aus Gewissensgründen folgt nun ein Urteilsauszug, der sich mit der Frage des Zurückbehaltungsrechtes aus $\$ 320$ BGB oder $\$ 273$ BGB beschäftigt.

\section{d. Urteil des AG Marburg v. 27. 6. 1980, Az.: yo C 197/80:}

...

II. Der Beklagte kann der Klageforderung nicht die Einrede des nicht erfüllten Vertrages nach $\$ 320$ BGB entgegenhalten, denn die Klägerin hat die aus den Stromlieferungsverträgen resultierende Hauptleistungsverpflichtung, dem Beklagten mangelfreie Energie in Form von elektrischem Strom zur Verfügung zu stellen, unstreitig erfüllt.

III. Ein Zurückbehaltungsrecht läßt sich auch nicht aus $\$ 273$ BGB herleiten.

Nach dieser Bestimmung darf derjenige, der aus demselben Rechtsverhältnis, auf dem seine eigene Verpflichtung beruht, einen fälligen Anspruch gegen den Gläubiger hat, die ihm obliegende Leistung verweigern, bis der Gläubiger geleistet hat. Auch die Verletzung einer vertraglichen Nebenpflicht kann einen solchen Gegenanspruch unter dem rechtlichen Gesichtspunkt einer positiven Forderungsverletzung begründen.

I. Die Klägerin hat keine Hinweispflicht verletzt, die einen zur Leistungsverweigerung berechtigenden Gegenanspruch des Beklagten begründet.

a) In der Rechtsprechung und Literatur zum Problem des Teilzahlungsboykotrs bei der Lieferung von Atomstrom wird nahezu ausschließlich die Ansicht vertreten, Gegenstand des Stromliefervertrages sei lediglich die Lieferung und Bezahlung des abgenommenen Stroms, nicht aber die Art und Weise der Stromproduktion (so ausdrücklich: AG Hamburg NJW 1979, 231 5; AG Stuttgart NJW 1980, 1 108; Lüke NJW 1979, 2049; so wohl auch Schwerdtner JZ 1979, 810; a. A. AG Stuttgart NJW 1979 aaO; wohl auch Knieper KJ r 98077 ff.).

b) Selbst wenn man diese Auffassung als zu eng ansehen sollte, führt dies vorliegend zu keinem anderen Ergebnis.

Zwar ist es richtig, daß im Zuge des raschen Wandels und der zunehmenden Komplexität der rechtlichen, wirtschaftlichen, sozialen und technischen Verhältnisse die Bedeutung vertraglicher Nebenpflichten in besonderem Maße zugenommen und in einer stärkeren Verrechtlichung des Postulats der Rücksichtsnahme auf den Vertragspartner seinen Ausdruck gefunden hat (so ausdrücklich: Köhler in Staudinger, Kommentar zum BGB, r2. Aufl., $\$ 433$ Rdnr. 48 ff.; vgl. z. B. BGHZ 64, 46,49 m. w. N.)

\footnotetext{
7 Die Prakrikabilität dieses Vorschlags zur Obliegenheitserfüllung beweist ein niederländisches Beispiet: dort hat es - dem deutschen Strobo ähnlich - einen - Boykott - der sog. Kalkar Stever, den von den Stromunternehmen umgelegten niederländischen Beitrag für den Schnellen Brüter in Kalkar, gegeben, worauf es zu einem von den EVU ausgehenden Kompromiß kam: den Boykorteuren wurde Gelegenheit geboten, den Anteil für die sog. Kalkar-Steuer auf ein Sonderkonto zur Erforschung sanfter Energietechnologien zu entrichten. Vgl. hierzu Wolfgang Herte, Erfahrungen aus ähnlichen Kampagnen, in: Kein Atomkraftwerk mit unserem Geld, aaO, S. $\{2$ f.
} 
Die danach begründbare verragliche Prüfungs- und Aufklärungsverpflichtung des Herstellers und Händlers bezieht sich jedoch immer auf den konkreten Leistungsgegenstand und seine konkrete Verwendung durch den Verbraucher. Dagegen sind die Modalitäten seiner Herstellung der Disposition des Herstellers überlassen (vgl. Knieper KJ 1980, 77, 81). Eine Gefährlichkeit der Ware Strom als solcher wird jedoch vom Beklagten selbst nicht behauptet.

c) Hinzu kommt in diesem Zusammenhang ein Weiteres: Zweck einer Hinweispflicht kann es doch nur sein, dem Betroffenen, d. h. dem Beklagten - und nur um Pflichtverletzungen gerade $i \mathrm{hm}$ gegenüber geht es hier - Klarheit über die ihm unter Umständen drohende Gefahr zu verschaffen (vgl. BGHZ 64, 46, 49). Daraus folgt aber zugleich, daß demjenigen gegenüber, der über die Gefahren des fraglichen Produkts informiert ist wie der BekJagte, keine Hinweispflichtverletzung begangen werden kann.

2. Die Klägerin hat auch keine Fürsorgepflicht verletzt, die einen zur Leistungsverweigerung berechtigenden Gegenanspruch begründen könnte.

a) Die Klägerin verletzt keine aus $\$ 6$ EnWG in Verb. mit der Präambel des Gesetzes fließende Fürsorgepflicht. Dabei kann dahinstehen, ob Stromlieferungsverträge dem Kaufvertragsrecht angehören, oder ob - wie der Beklagte meint - bezüglich des Umfangs der Fürsorgepflichten $\$ 618$ Abs. 3 BGB entsprechende Anwendung finden muß. Soweit die Bestimmung des $\$ 6$ EnWG dem Beklagten überhaupt einen klagbaren Anspruch gewährt, ist dieser öffentlichrechtlicher Natur und richtet sich ausschließlich auf Anschluß und Versorgung aus dem Versorgungsnetz (vgl. Eiser-Riederer, Energiewirtschaftsrecht, Kommentar, $\$ 6$ Anm. 6). Einen weitergehenden Anspruch gewährt das EnWG nicht.

b) Das Amtsgericht Stuttgart (NJW 1979 aaO) hat die Ausdehnung der vertraglichen Nebenpflichten über den Bereich der gelieferten Ware hinaus insbesondere mit der durch die Produktion von Atomstrom entstehenden Lebensbedrohung für die Beklagten und die Bevölkerung allgemein gerechtfertigt. Im Unterschied zu diesem vom Amtsgericht Stuttgart entschiedenen Fall stellt die Klägerin dieses Rechtsstreits jedoch keinen Atomstrom her; vielmehr besteht ihre Aufgabe und tatsächliche Tätigkeit nur darin, daß sie den eingekauften Strom im Stadtgebiet den Endabnehmern zur Verfügung stellt. Dabei mag durchaus unterstellt werden, daß die Klägerin weiß, daß der von ihr gelieferte Strom auch in Atomkraftwerken gewonnen wird. Die vertraglichen Fürsorgepflichten auf Grund des Stromlieferungsvertrages beinhalten jedoch nicht, daß die Klägerin keinen derart gewonnenen Strom liefern oder die Bezahlung des entsprechenden Anteils an Atomstrom - soweit dieser spezifizierbar wäre verweigern dürfte bzw. müßte. Dieses Verlangen an die Klägerin versetzte sie vielmehr in eine Situation, in der sie die Erfüllung ihrer Hauptleistungsverpflichrungen aus den Stromlieferungsverträgen, nämlich die Lieferung von ausreichendem und möglichst preisgünstigem Strom, unter Umständen nicht mehr gewährleisten könnte.

c) Mit der Argumentation, die Klägerin treffe letztlich die gleichen Fürsorgepflichten, wie die Energieversorgungsunternehmen, bewegt sich der Beklagte auf einer politischen Ebene, die rechtlich jedenfalls nicht haltbar ist. Angesichts der von ihm aufgezeigten engen gesellschaftlichen Zusammenhänge zwischen Stromproduktion und -lieferung durch Energieversorgungsunternehmen, Verteilung, Lieferung und Tarifgestaltung durch die Klägerin und Verbrauch durch die Endabnehmer mag dem Beklagten die juristisch notwendige Begrenzung allein auf das Vertragsverhältnis zwischen ihm und der Klägerin und der aus diesem Vertrag resultierenden Nebenpflichten als verkürzt erscheinen. Das BGB und die ZPO gehen aber vom Modell des zwischen Privatpersonen zu lösenden sozialen Konflikts aus (vgl. Rasehorn, ZRP 1980, 6). Die über das synallagmatische Vertragsverhältnis hinausgehende tiefgreifende soziale Problematik zwischen Angewiesensein auf die Lieferung von Strom einerseits und Ausgeliefertsein an eine als lebensbedrohend erlebte Qualität der Forschung andererseits ist daher nach Uberzeugung des Gerichts mit den Mitteln des materiellen und Zivilprozeßrechts nicht operationabel zu machen.

3. Schließlich muß hinsichtlich der behaupteten Gegenansprüche aus positiver Forderungsverletzung noch klargestellt werden, daß zur dogmatischen Begründung eines derartigen Anspruchs - sieht man von der Frage des Verschuldens ab - auch die Behauptung und gegebenenfalls der Beweis eines von der Klägerin verschuldeten konkreten Schadens gehören (so zutreffend Lüke aaO). Der Beklagte argumentiert zwar mit einem Schadensbegriff, meint jedoch letztlich Gefährdung. Eine allgemeine Haftung für Gefährdungen gewährleistet das Rechtsinstitut der positiven Forderungsverletzung nicht.

Beachtlich an dieser Entscheidung ist zunächst die eingehende Beschäftigung mit den Argumenten des Stromzahlungsboykotteurs. Das ist entgegen der Annahme 
Gcrlachs ${ }^{8}$ keineswegs üblich. Auffällig und insoweit symptomarisch ist jedoch die Hilflosigkeit des Gerichts angesichts der Dimension des zu lösenden Konflikres. Unter Berufung auf die eingeschränkte Sicht von $Z P O$ und $B G B$ wird eine Konfliktlösung mit juristischen Mitreln für unmöglich erklärt. Dogmatische Schwierigkeiten wurden erkannt, doch fehlt es noch an jedem Versuch zu ihrer Bewältigung. Die Tatsache etwa, daß lediglich eine konkrete Gefährdung und noch kein konkreter Schaden vorliegt, führt nach bisherigem dogmatischem Verständnis zum Ausschluß jeglichen Gegenanspruchs. Könnte aber nicht dann etwas anderes gelten, wenn der drohende Schaden in seinem katastrophalen Ausmaß jede spätere Streitschlichtung unmöglich machen würde?"

Abschließend seien noch die neueren prozessualen Tendenzen erwähnt. Die EVU gehen zunehmend dazu über, nicht nur die einbehaltenen Stromgelder einzuklagen, sondern begehren auch folgende drei Feststellungen: I) ein Leistungsverweigerungsrecht seitens des Stromzahlungsboykotteurs bestehe nicht, 2) das EVU sei im Falle weiterer Rechnungskürzungen zur Stromabschaltung und 3) zur fristlosen Kündigung des Liefervertrages berechtigt. Die Gründe für diese Feststellungsanträge werden darin zu suchen sein, daß die EVU bestrebt sind, alle Urteile berufungsfähig zu machen, damit kein - neue Wege der Zivilrechtsdogmatik beschreitender Amtsrichter endgültig über den Rechtsstreit entscheiden kann ${ }^{\text {to }}$, und schließlich darin, daß damit die Konsequenzen für die Stroboteilnahme wegen des höheren Streitwertes sclion auf der Prozeßkostenebene härter sind. Die mit diesen Feststellungsanträgen zusammenhängenden juristischen Fragen, namentlich das Feststellungsinteresse betreffend, sind komplex. Vom Amesgericht Köln beispielsweise wurden diese Fragen sehr unterschiedlich beurteilt. Auf den Entscheidungsabdruck kann hier jedoch verzichtet werden, weil drei dieser Urteile leicht zugänglich sind." Das LG Köln, hat mit Beschluß vom 2. 4. 198I, Az. I S 32/8I, dazu folgendes ausgeführt:

Das Gericht weist darauf hin, daß Bedenken gegen das Bestehen eines Feststellungsinteresses für die Berufungsanträge bestehen, weil sich die Voraussetzungen, unter denen die Klägerin gegebenenfalls künftig zur Sperrung oder Kündigung des Vertragsverhältnisses berechrigt ist, nicht im voraus festlegen lassen.

Wenn diese Linie der Interpretation gehalten werden kann ${ }^{12}$, bleibt auch die juristische Auseinandersetzung um den Stromzahlungsboykott weiterhin offen.

Willi Gross

8 Gerlach, aaO S. 366, lagen nur einige wenige Entscheidungen zur Beureilung vor.

9 Hierauf kann im Rahmen der Dokumentation nicht näher eingegangen werden. Zu den Möglichkeiten, die rechtliche Argumentation weiter zu entwicklen vgl. aber insgesamt Knieper, aaO S. $77 \mathrm{ff}$.

10 Gegen das in Fn. 3) erwähnte Urteil des AG Stuttgart war jedenfalls ein Rechtsmittel nicht möglich. Die Versuche von Lüke (NJW 1979, S. 2049 f.) ein solches zu konstruieren muten gekünstelt an.

I Abgedruckt in der Zeitschrift Wohnungswirtschaft und Mietrecht $198 \mathrm{I}, 5$. $88 \mathrm{ff}$. (Heft 4 )

12 Das Landgericht Dortmund, NJW 198 I, S. 764 f., hat bereits eine andere Auffassung vertreten, die mit Urteil des OLG Hamm v. 1.7. 1981, Az. 8 U 19/81, bestätigt wurde. 


\section{Buchbesprechungen}

Ulrich K. Preuss, Die Internalisierung des Subjekts. Zur Kritik der Funktionsweise des subjektiven Rechts Frankfurt/Main 1980 (Subrkump Verlag), 356 Seiten, DM 30,-

I.

Die Legalität wird zunehmend zu einer politischen Verkehrsform, die lediglich noch ihre eigene Außerkraftsetzung zu legitimieren vermag, dieses Resumee seiner 1973 publizierten rechtstheoretischen Studie "Gesellschaftliche Bedingungen der Legalizät $\alpha^{\prime}$ antizipierte eine verfassungsstrukturelle Entwicklung, die unter den Stichworten der Normalisierung des Ausnahmezustandes bzw. des ralltäglichen Notstandes، als das entscheidende Charakteristikum der Veränderung der Formen politischer Herrschaft in der Bundesrepublik insbesondere in der zweiten Hälfte der joer Jahre Schlagzeilen machte.

In Preuß jüngster Monographie aus dem Jahr 1979 mit dem geheimnisvollen Obertitel -Die Internalisierung des Subjekts* fällt die Diagnose über Zustand und Entwicklungsperspektive der Gesellschaft sogar noch deutlich schärfer, katastrophentheoretisch radikalisierter aus. Danach haben sich alle drei elementaren Vernunftkategorien, um die das frühliberale Konzept einer rationalen, seiner eigenen Geschichte und Geschicke mächtigen Vergesellschaftung zentriert war, nämlich Individuum (Subjekt), Recht und Staat im Entfaltungsprozeß der bürgerlichen Gesellschaft zur Irrationalität hin aufgelöst: In dem Maße, wie das Legalitätssystem weitgehend durch andere Strategien und Mechanismen gesellschaftlicher Steuerung überlagert,

I In: Legalitat und Pluralismus, Beiträge zum Verf́assungsrecht Jer Bundesrepublik Deutschland, Frankfurr/M. 1973, S. 109 ff. $(112 \mathrm{f})$ zersetzt und aufgelöst worden und damit wdie Konstituierung einer sozialen Ordnung durch Recht der Idee nach aufgegeben worden ist « $(258)$, unterliegen auch das individuelle wie das gesellschaftliche Subjekt (Individuum und Staat) grundlegenden Veränderungen, die zu einer gänzlich veränderten Konstruktion von Subjektivität und politischer Herrschaft sowie des Verhältnisses beider zueinander geführt haben.

Zentral geht es dabei nach Preuß um einen parallelen Prozeß des Verlusts an Souveränität, der in gleicher Weise zur Zerstörung der Autonomie des Individuums wie zur Schwächung der "ordnungspolitischen Kraft * (257) des Gesetzgebers geführt hat. Nicht nur das Individuum wird unter die "Regelmäßigkeiten und Funktionsimperative der gesellschaftlichen Ordnung « (250) gezwungen, sondern auch der Gesetzgeber wird an die * Bewahrung einer inhaltsvollen Identitär des Gemeinwesens « (250) gebunden; für seinen *verlorengegangenen Eigenwert (271) wird das individuelle Subjekt durch - ihrerseits monetär konvertierbare - staatliche Kompensationsleistungen entschädigt, windividuelle Identität (wird) durch etwas definiert, was austauschbar ist « (272) mit der Konsequenz, daß das wsubjektive Widerstandspotential, das die biographische Einmaligkeit jedes Individuums in die kollektive Selbstreproduktion der Gesellschaft einbringt, vollkommen ausgelöscht (ist)* (329). Ebensowenig wie das Individuum »biographische Einmaligkeit « unter gesellschaftlichen Bedingungen auszubilden vermag, unter denen -ihm sein ökonomischer und sozialer Wert gewissermaßen verordnet wird (270), ebensowenig ist das System politischer Herrschaft in der Lage, den gesellschaftlichen Zusammenhang bewußt zu gestalten und ihm dadurch historische Einmaligkeit zu verleihen. $\mathrm{Zwar}$ wird das politisch-administrative 